\title{
THE
}

\section{Impact of the Reduced Drag Coefficient on Ocean Wave Modeling under Hurricane Conditions}

\author{
II-Ju Moon \\ Isaac Ginis \\ University of Rhode Island, iginis@uri.edu \\ Tetsu Hara \\ University of Rhode Island, thara@uri.edu
}

Follow this and additional works at: https://digitalcommons.uri.edu/gsofacpubs

\section{Citation/Publisher Attribution}

Moon, I., Ginis, I., \& Hara, T. (2008). Impact of the Reduced Drag Coefficient on Ocean Wave Modeling under Hurricane Conditions. Monthly Weather Review, 136, 1217-1223. doi: 10.1175/2007MWR2131.1 Available at: https://doi.org/10.1175/2007MWR2131.1

This Article is brought to you for free and open access by the Graduate School of Oceanography at DigitalCommons@URI. It has been accepted for inclusion in Graduate School of Oceanography Faculty Publications by an authorized administrator of DigitalCommons@URI. For more information, please contact digitalcommons-group@uri.edu. 
NOTES AND CORRESPONDENCE

\title{
Impact of the Reduced Drag Coefficient on Ocean Wave Modeling under Hurricane Conditions
}

\author{
IL-Ju MoON \\ College of Ocean Science, Cheju National University, Jejusi, South Korea \\ IsAaC Ginis AND Tetsu HARA \\ Graduate School of Oceanography, University of Rhode Island, Narragansett, Rhode Island
}

(Manuscript received 2 January 2007, in final form 22 June 2007)

\begin{abstract}
Effects of new drag coefficient $\left(C_{d}\right)$ parameterizations on WAVEWATCH III (WW3) model surface wave simulations are investigated. The new parameterizations are based on a coupled wind-wave model $(\mathrm{CWW})$ and a wave tank experiment, and yields reduced $C_{d}$ at high wind speeds. Numerical experiments for uniform winds and Hurricane Katrina (2005) indicate that the original $C_{d}$ parameterization used in WW3 overestimates drag at high wind speeds compared to recent observational, theoretical, and numerical modeling results. Comparisons with buoy measurements during Hurricane Katrina demonstrate that WW3 simulations with the new $C_{d}$ parameterizations yield more accurate significant wave heights compared to simulations with the original $C_{d}$ parameterization, provided that accurate high-resolution wind forcing fields are used.
\end{abstract}

\section{Introduction}

Accurate forecasts of extreme wind waves associated with hurricanes are of great importance for minimizing the loss of life and property in maritime and coastal areas. In recent years considerable efforts have been made to improve the skill of ocean wave modeling under hurricane conditions (Moon et al. 2003, hereafter M03; Chao et al. 2005, hereafter C05; Tolman et al. 2005, hereafter T05; Tolman and Alves 2005, hereafter TA05), but several issues still remain unresolved. One of the major issues is the difficulty in producing accurate high-resolution wind inputs that resolve rapidly varying wind structure in time and space near the hurricane core. In previous studies dealing with wave simulations, hurricane wind fields were computed either empirically, by using surface wind data at fixed radii from the storm center in four quadrants provided by the $\mathrm{Na}$ -

Corresponding author address: Il-Ju Moon, College of Ocean Science, Cheju National University, Aradong, Jejusi, Jejudo 690756, South Korea.

E-mail: ijmoon@cheju.ac.kr tional Hurricane Center (NHC; M03), or directly, by computation from global atmospheric or hurricane prediction models (C05; T05). Because of the insufficient spatial resolution, these methods have limitations in describing detailed and accurate wind structures and often yield underestimated wind forcing near the hurricane's eyewall (C05; T05).

Another important issue is accuracy in parameterization of wave growth for high wind speeds inside the wave model. Wave growth rates scale with the corresponding friction velocity or drag coefficient (Tolman and Chalikov 1996). The present drag coefficient $\left(C_{d}\right)$ parameterization used in the operational WAVEWATCH III (WW3) model is based on observations in low to moderate wind conditions and yields increased $C_{d}$ with increasing wind speed (WAMDI Group 1988; Tolman 2002). However, recent observational, laboratory, theoretical, and modeling studies suggest that $C_{d}$ ceases to increase with wind speed for high wind speeds (Powell et al. 2003; Donelan et al. 2004, hereafter D04; Emanuel 2003; Moon et al. 2004a,b,c). Recently, a new $C_{d}$ parameterization has been suggested based on a coupled wind-wave model (CWW; Moon et al. 2007), 
which also shows that the neutral $C_{d}$ levels off under hurricane winds.

As demonstrated in Moon et al. (2004b), the operational WW3 significantly overestimates $C_{d}$, and hence overestimates wave growth rates at high winds. The effect of the overestimated drag on wave growth leads to overestimation of simulated wave heights ( $\mathrm{C} 05$; T05; TA05). However, most wave forecast models demonstrate reasonable performance for high winds, apparently resulting from the mutual compensation of underestimated wind input and the overestimated surface drag.

The main objective of this study is to investigate the impacts of the reduced $C_{d}$ on WW3 wave simulations under high winds and hurricane conditions using the $C_{d}$ parameterizations of Moon et al. (2004a) and D04. To reduce biases caused by inaccurate wind input, highresolution wind fields are produced using the Hurricane Research Division (HRD) tropical cyclone observing system (Powell et al. 1998) from the National Oceanic and Atmospheric Administration (NOAA). WW3 simulations are performed using both the operational and new $C_{d}$ parameterizations under uniform winds and hurricane winds.

A brief outline of the WW3 model and the parameterizations of $C_{d}$ are introduced in section 2. Sections 3 and 4 describe experimental designs and specifications of hurricane wind forcing, respectively. Results are discussed in section 5, and the summary and conclusion are given in the last section.

\section{Parameterization of the drag coefficient in WW3}

WW3 is a third-generation surface wave model that has been extensively validated for global and regional wave forecasts (Tolman 2002; T05). The drag coefficient parameterization in WW3 is based on Tolman and Chalikov (1996), in which $C_{d}$ at a given reference height (here, $10 \mathrm{~m}$ ) is expressed as

$$
C_{d}=10^{-3}\left(0.021+\frac{10.4}{R^{1.23}+1.85}\right)
$$

with

$$
R=\ln \left(\frac{10 g}{\chi \sqrt{\alpha} u_{\mathrm{e} 10}^{2}}\right),
$$

where $\chi$ is a constant value (0.2), $u_{\mathrm{e} 10}$ is a effective wind speed at $10-\mathrm{m}$ height, and $\alpha$ is the nondimensional energy level at high frequencies, which is expressed in terms of the wave age $\left(c_{p} / u_{*}\right)$,

$$
\alpha=0.57\left(\frac{c_{p}}{u_{*}}\right)^{-3 / 2} .
$$

Here, $u_{*}$ is the friction velocity and $c_{p}$ is the phase speed at the peak frequency. For fully grown seas this parameterization is similar to the commonly used bulk parameterization with a constant Charnock coefficient. However, it yields higher drag for younger waves at any given wind speed, and is consistent with the empirical parameterizations by Donelan (1990) and Drennan et al. (2003).

In this study we investigate how the predicted wave field is modified when the wind input parameterization in WW3 is replaced by the new $C_{d}$ suggested by Moon et al. $(2004 a, b)$ and D04. In the CWW of Moon et al. $(2004 a, b)$ the complete wave spectrum is first constructed by merging the WW3 spectrum in the resolved frequency range (vicinity of the spectral peak) with the spectral tail parameterization of Hara and Belcher (2002). The result is then incorporated into the wave boundary layer model (Hara and Belcher 2004) to explicitly calculate the wave-induced stress vector, the mean wind profile, and $C_{d}$ over any given complex seas. This $C_{d}$ is used in the wind input parameterization at the next time step in the WW3.

\section{Experimental design}

Two kinds of experiments are designed to investigate the effect of three different parameterizations of $C_{d}$ (original, CWW, and D04 parameterizations) on wave modeling. First, idealized experiments under spatially homogeneous winds from 10 to $60 \mathrm{~m} \mathrm{~s}^{-1}$ are performed, assuming that northward winds blow over the model domain of $3000 \mathrm{~km} \times 1500 \mathrm{~km}$ (in latitude and longitude direction) with 2000-m water depth. Second, real-case experiments for Hurricane Katrina (2005) are conducted. For real-case experiments, the results of three wave simulations using the original, $\mathrm{CWW}$, and D04 wind input parameterizations are compared. As described earlier, because the three sets of experiments are identical, except for using different $C_{d}$ parameterizations, the wave model parameters used in all of the experiments are identical, as follows: $1800 \mathrm{~s}$ (time step and wind input interval), 24 directions (directional resolution), and $1 / 12^{\circ} \times 1 / 12^{\circ}$ (spatial grid resolution). The wave spectrum is discretized using 40 frequencies extending from 0.0285 to $1.1726 \mathrm{~Hz}$ (a wavelength of $1.1-1920 \mathrm{~m})$, with a logarithmic increment $f_{n+1}=1.1 f_{n}$, where $f_{n}$ is the $n$th frequency. Simulated significant wave heights and input winds are compared with measurements from the National Data Buoy Center (NDBC) for validation. 

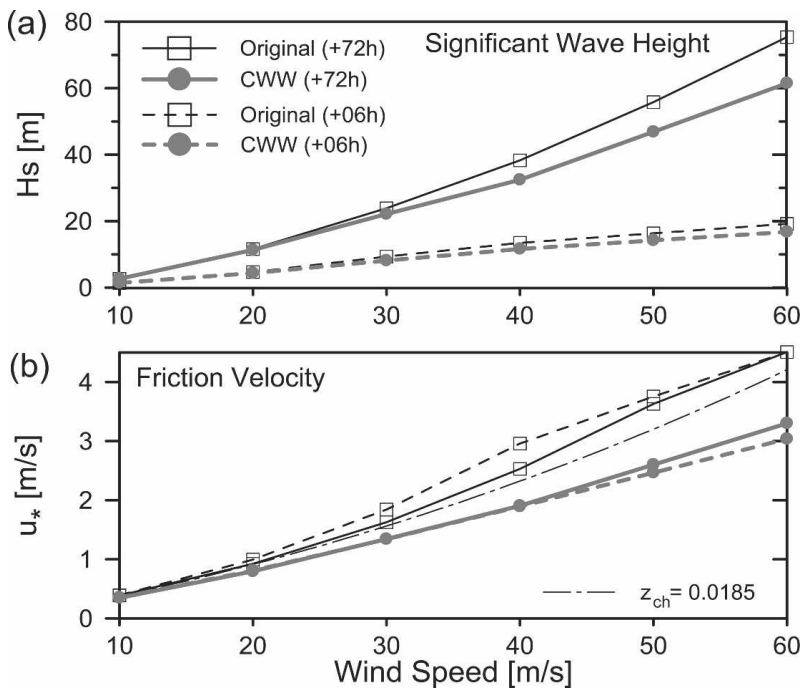

FIG. 1. (a) Significant wave heights and (b) friction velocity vs wind speed at $10 \mathrm{~m}$ estimated using the CWW (thick gray lines) and original (thin black lines) parameterizations in WW3 at 6 (dashed lines) and 72 (solid lines) $\mathrm{h}$ after the onset of the uniform constant winds from 10 to $60 \mathrm{~m} \mathrm{~s}^{-1}$.

\section{Hurricane wind field specification}

High-resolution hurricane surface wind fields in this study are produced using the HRD tropical cyclone observing system (Powell et al. 1998). The HRD winds are routinely provided at interval of every 3 or $6 \mathrm{~h}$, with the spatial resolution of about $6 \mathrm{~km} \times 6 \mathrm{~km}$, covering an area of about $8^{\circ}$ latitude $\times 8^{\circ}$ longitude around the hurricane's center. The wind data in gridded form are available from the HRD Web site for all hurricanes in the Atlantic basin since 1994 (online at http://www. aoml.noaa.gov/hrd/data_sub/wind.html). The following four steps are taken to construct input wind fields into WW3: First, for every HRD wind snapshot, radial wind profiles are calculated at a $5^{\circ}$ interval around the hurricane center. Second, the radial wind profiles are interpolated from 3 (or 6)-h intervals to 30-min intervals. Third, the locations of hurricane's center are interpolated at 30-min intervals. Fourth, two-dimensional wind fields are generated by spatial (azimuthal) interpolation of the radial profiles. This method yields more accurate hurricane wind fields than those produced using the $\mathrm{NHC}$ wind data at a few fixed radii in four quadrants of the hurricane (M03). The computed winds for Hurricane Katrina are compared with measurements in the next section.

\section{Results and discussions}

The idealized experiment with spatially homogeneous winds has been performed using the original and

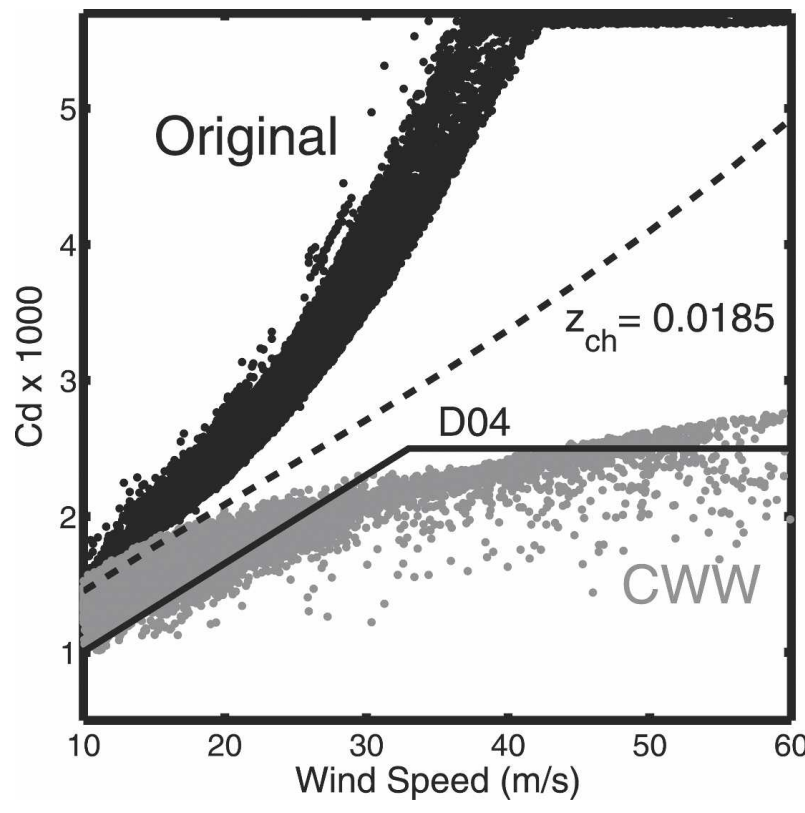

FIG. 2. A scatterplot of $C_{d}$ as a function of the wind speed at 10-m height obtained using CWW (gray dots) and original (black dots) parameterizations for Hurricane Katrina. Model outputs at all grid points every $6 \mathrm{~h}$ are used for this scatterplot. The dashed line represents a constant Charnock coefficient $\left(z_{\mathrm{ch}}=0.0185\right)$; a solid line represents a parameterization based on D04.

CWW wind input parameterizations. In the experiment, a central point at the northern part of the domain, where the effect of the model boundaries is negligible, is selected to obtain the mean wave parameters. Simulated significant wave height $\left(H_{s}\right)$ at different wind speeds are presented after 6 and $72 \mathrm{~h}$ from the onset of wind (Fig. 1a). For $10 \mathrm{~m} \mathrm{~s}^{-1}$ wind speed, $H_{s}$ becomes constant within $72 \mathrm{~h}$, indicating that the wave field becomes fully developed by that time. For higher wind speeds the wave fields are still developing after $72 \mathrm{~h}$. The figure shows that after $72 \mathrm{~h}$ (solid lines) the difference in $H_{s}$ between the two $C_{d}$ parameterizations becomes larger with increased wind speed, reaching $15 \mathrm{~m}$ at a wind speed of $60 \mathrm{~m} \mathrm{~s}^{-1}$. This is mainly attributed to the reduced $C_{d}$ (or friction velocity, $u_{*}$ ) with the CWW parameterization at high wind speeds (Fig. 1b). As reported by Moon et al. (2004a), at high wind speeds the CWW predicts lower drag as waves become younger, while the original parameterization predicts higher drag for young seas. At $6 \mathrm{~h}$ (dashed lines), the difference of $H_{s}$ is relatively small (less than $3 \%$ reduction) even if the difference of $u_{*}$ is significant (up to $30 \%$ reduction). This is likely because the accumulated effect of the reduced $u_{*}$ is still small at this stage.

The scatterplot of $C_{d}$ in the real-case experiment for Hurricane Katrina (2005) is shown in Fig. 2. At high winds under the hurricane where young waves are dom- 
Swath of Significant Wave Height (Hurricane Katrina)

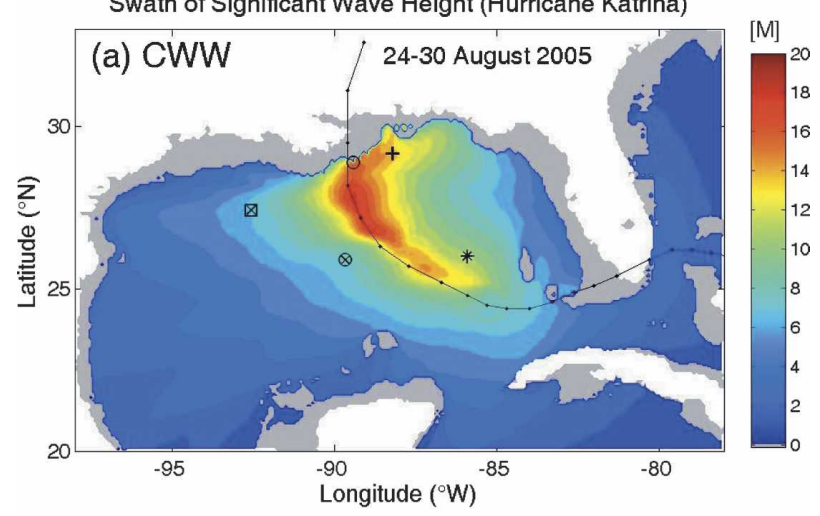

Swath Difference between original and CWW in Hs

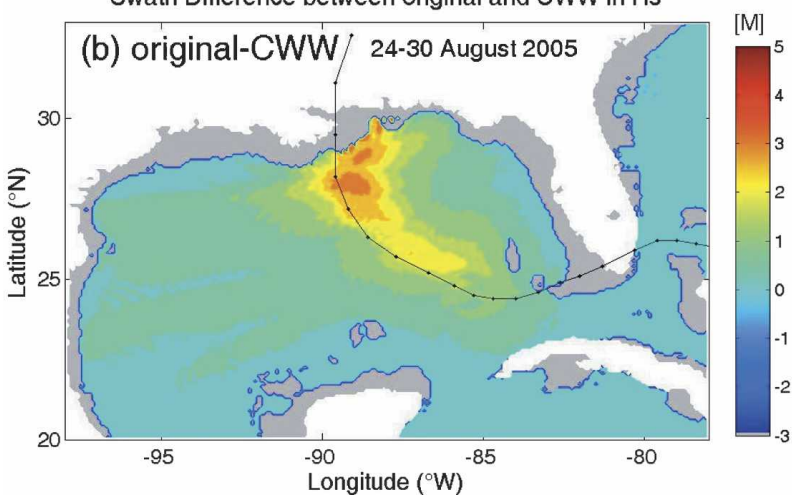

FIG. 3. (a) Swath of $H_{s}$ produced by WW3 using CWW parameterization and (b) the difference of the swath of $H_{s}$ between $\mathrm{CWW}$ and the original parameterizations during the passage of Hurricane Katrina. A solid line with dots is the storm track. Symbols represent locations of buoys (plus sign: buoy 42040, boxed crisscross: buoy 42038, asterisk: buoy 42003, and circled crisscross: buoy 42001) and C-MAN stations (open circle: BURL1). Gray colors represent shallow-water seas below 30-m depth.

inant, CWW produces a much lower $C_{d}$, while the original parameterization predicts a much higher $C_{d}$. Results of CWW are similar to those of D04 based on the wave tank experiments. A constant Charnock coefficient $\left(z_{\mathrm{ch}}=0.0185\right.$, dashed line $)$, which is widely used in atmospheric models, lies between the two models. An interesting point is that the original parameterization sets an upper limit of $C_{d}$ (mostly at high wind speeds with young waves) for model stability. This fact further suggests that the original parameterization overestimates $C_{d}$ at high winds.

The swath pictures of $H_{s}$ for Katrina are shown in Fig. 3. The figure represents maximum values at each grid point throughout the hurricane passage. It is seen that the highest waves (about $16.5 \mathrm{~m}$ ) are found from the WW3 simulation using the CWW parameterization when the hurricane approaches shallow seas before making landfall (Fig. 3a). Larger differences between

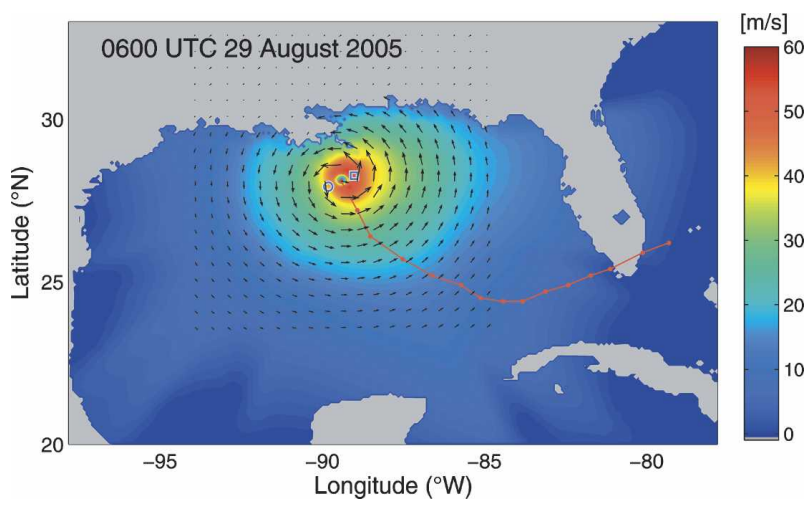

FIG. 4. A high-resolution surface wind field of Hurricane Katrina produced using the HRD tropical cyclone observing system at 0600 UTC 29 Aug 2005. Square and circle represent positions for comparisons of sea surface directional wave spectra.

the two models (original - CWW) appear to the right of the hurricane along its track. The difference is as large as $3 \mathrm{~m}$ near the region where the highest waves occur (Fig. 3b). In this figure, results at shallow-water seas below 30-m depth are removed because of limitations of the resolving depth in the wave model.

When the difference is the largest (0600 UTC $29 \mathrm{Au}$ gust), sea surface directional wave spectra are compared at the right and left points (see Fig. 4) along the radius of the maximum wind speed (RMW), as seen in Fig. 5. The spectral shapes produced from three parameterizations (original, CWW, and D04) appear to be similar, that is, the wave spectra to the right of the hurricane are narrow; the wave spectra to the left are wide and spreading. But, the simulated peak wavenumber and significant wave height from the original model is much higher than those from the CWW and D04 parameterization, probably due to the overestimated $C_{d}$. In particular, the difference is larger to the right of hurricane than to the left. Overall results from the CWW and D04 are very similar.

The model-simulated $H_{s}$ and winds are compared with measurements at four buoys and one CoastalMarine Automated Network (C-MAN) station (see Fig. 3a) along the track of Hurricane Katrina (Table 1 and Fig. 6). The comparison of winds (Fig. 6a) shows that the computed and observed speeds/directions are in very good agreement with the root-mean-square (rms) errors of $1.74 \mathrm{~m} \mathrm{~s}^{-1}$ in speed and $11^{\circ}$ in direction. With this accurate wind forcing, the WW3 simulation at buoy 42040 (Fig. 6b) with the original $C_{d}$ parameterization overestimates $H_{s}$ by about $1.5 \mathrm{~m}$ throughout the hurricane passage, while the simulation with the CWW and D04 $C_{d}$ parameterization yields much reduced errors. The comparison at all five measurement locations also shows significant improvement (Table 1). 

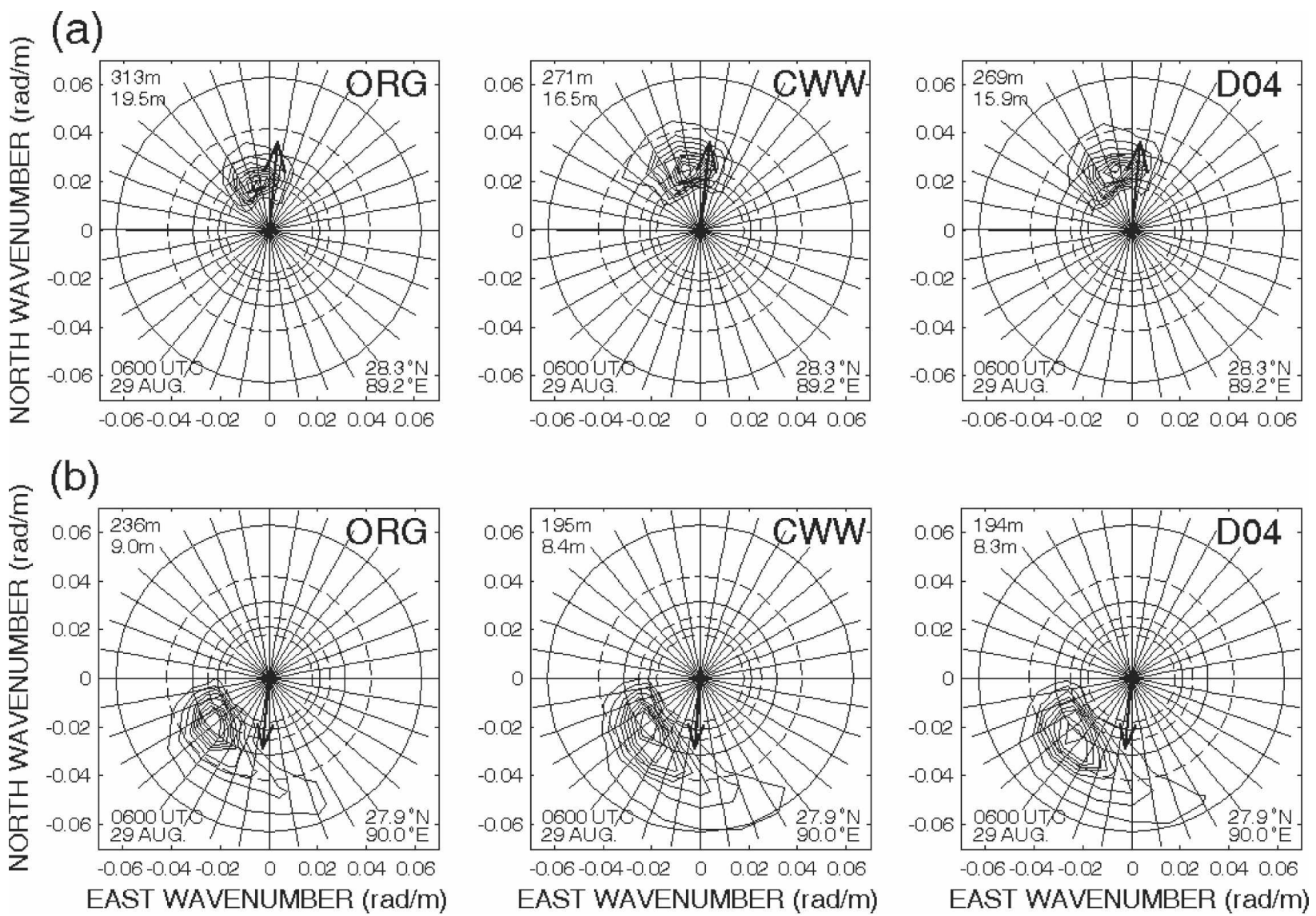

FIG. 5. Sea surface directional wave spectra produced by WW3 using (left) original, (middle) CWW, and (right) D04 $C_{d}$ parameterization at the right and left points along the RMW at 0600 UTC 29 Aug 2005 (see Fig. 4). The dashed circles (from outer to inner) correspond to wavelengths of 150, 250, and $350 \mathrm{~m}$; the solid circles indicate wavelengths of 100, 200, and $300 \mathrm{~m}$. Each spectrum contains nine contours, linearly spaced from $10 \%$ to $90 \%$ of the peak spectral density. The peak wavelengths and the significant wave height are shown in the upper-left corner of each spectrum. The thick arrows extend in the downwind direction with their length proportional to the surface wind vectors at each point. A wind speed of $20 \mathrm{~m} \mathrm{~s}^{-1}$ corresponds to a length of $0.03 \mathrm{rad} \mathrm{m}^{-1}$. Observation time (bottom left) and location (bottom right) are shown in the spectrum.

From WW3 simulations during Hurricane Isabel, T05 found that underestimated wind input produced a realistic wave height near the hurricane core, likely because the drag coefficient was overestimated. This implies that wave heights would be overestimated when accurate wind fields were used in WW3. Our simulations indeed demonstrate that WW3 with the original $C_{d}$ parameterization forced by accurate wind fields overestimates the significant wave height, and that the combination of the accurate wind forcing and the new $C_{d}$ parameterizations yields more accurate wave fields.

\section{Summary and concluding remarks}

The present drag coefficient $\left(C_{d}\right)$ parameterization used in the WAVEWATCH III (WW3) model is based on observations in low to moderate wind conditions and yields increased $C_{d}$ with increasing wind speed. However, recent observational, laboratory, theoretical, and modeling studies for high wind speeds $\left(>30 \mathrm{~m} \mathrm{~s}^{-1}\right)$ suggest that $C_{d}$ ceases to increase with wind speed. Three recent publications (C05; T05; TA05) point out

TABLE 1. Mean and rms errors between buoy and models (original, CWW, and D04 parameterizations) for wind speed, wind direction, and significant wave heights. Comparisons are made at five locations along the track of Hurricane Katrina (Fig. 3a).

\begin{tabular}{llcr}
\hline \hline & & $\begin{array}{c}\text { Mean error } \\
\text { (model buoy) }\end{array}$ & Rms error \\
\hline Wind & Speed $\left(\mathrm{m} \mathrm{s}^{-1}\right)$ & 0.75 & 1.74 \\
& Direction ( $\left.{ }^{\circ}\right)$ & 1.84 & 10.94 \\
Significant wave & Original (m) & 1.51 & 1.71 \\
height & CWW (m) & 0.37 & 0.96 \\
& D04 (m) & 0.38 & 0.98 \\
\hline
\end{tabular}


(a)

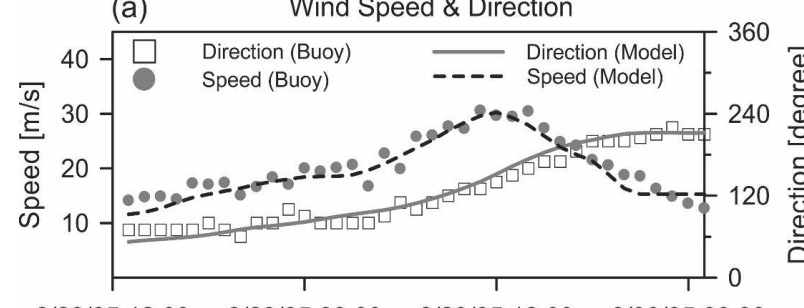

$8 / 28 / 0512: 00 \quad 8 / 29 / 0500: 00 \quad 8 / 29 / 0512: 00 \quad 8 / 30 / 0500: 00$

(b)

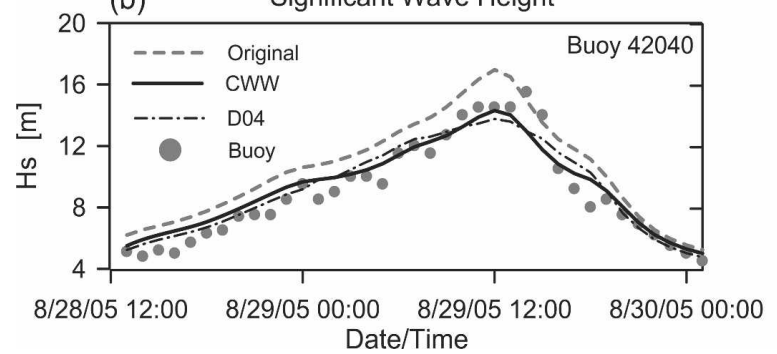

FIG. 6. Comparisons of (a) wind speed and direction, and (b) significant wave height between the model and buoy at buoy 42040 (see Fig. 3a). For the $H_{s}$ comparison, three results from the CWW, D04, and original parameterization are used.

that the overestimated $C_{d}$ used in WW3 may contribute to errors in operational wave forecasts at the National Centers for Environmental Prediction (NCEP), particularly at extreme wind events.

The present study investigated the impact of new $C_{d}$ parameterizations on WW3 simulations. The new parameterizations are based on the coupled wind-wave model (CWW; Moon et al. 2004a,b,c) and the wave tank experiment (D04), and yields reduced $C_{d}$ at high winds. The WW3 simulations were performed for uniform winds and Hurricane Katrina with both the original and the new $C_{d}$ parameterizations. The calculated $C_{d}$ with the original parameterization increases significantly as wind speeds increase, resulting in overestimation at high wind speeds, while the $C_{d}$ with the new parameterizations levels off at high winds, consistent with recent results (Powell et al. 2003; Emanuel 2003). With uniform wind forcing applied for $72 \mathrm{~h}$, the significant wave height is not affected at $10 \mathrm{~m} \mathrm{~s}^{-1}$ wind speed, but is reduced by as much as $15 \mathrm{~m}$ at wind speeds of $60 \mathrm{~m} \mathrm{~s}^{-1}$ in the CWW parameterization. For Hurricane Katrina, high-resolution wind fields from the HRD tropical cyclone observing system were utilized. The largest decrease $(3 \mathrm{~m})$ in $H_{s}$ appears to the right of the hurricane track where the highest waves occur. Comparisons with measurements show that the CWW and D04 parameterizations yield more accurate $H_{s}$ than the original $C_{d}$ parameterization, provided that accurate high-resolution wind forcing is used.

This study demonstrates that the combination of the new $C_{d}$ parameterizations and the accurate highresolution wind forcing may greatly improve the prediction of surface waves in hurricane conditions. However, only the one hurricane case has been investigated in this study. Effects of the reduced $C_{d}$ on WW3 may appear differently in other hurricane cases. More realcase experiments will be necessary to substantiate the results reported here.

Acknowledgments. This work was supported by the U.S. National Science Foundation through Grant ATM0406895. TH and IG also thank the U.S. Office of Naval Research (CBLAST Program Grant N00014-0610729) for additional support. Il-Ju Moon was supported by the Korea Meteorological Administration Research and Development Program under CATER 2006-2301 completely.

\section{REFERENCES}

Chao, Y. Y., J. H. G. M. Alves, and H. L. Tolman, 2005: An operational system for predicting hurricane-generated wind waves in the North Atlantic Ocean. Wea. Forecasting, 20, 652-671.

Donelan, M. A., 1990: Air-sea interaction. The Sea, B. Le Mehaute and D. M. Hanes, Eds., Ocean Engineering Science, Vol. 9B, Wiley and Sons, 239-292.

—, B. K. Haus, N. Reul, W. J. Plant, M. Stiassnie, H. C. Graber, O. B. Brown, and E. S. Saltzman, 2004: On the limiting aerodynamic roughness of the ocean in very strong winds. Geophys. Res. Lett., 31, L18306, doi:10.1029/2004GL019460.

Drennan, W. M., H. C. Graber, D. Hauser, and C. Quentin, 2003: On the wave age dependence of wind stress over pure wind seas. J. Geophys. Res., 108, 8062, doi:10.1029/2000JC000715.

Emanuel, K. A., 2003: A similarity hypothesis for air-sea exchange at extreme wind speeds. J. Atmos. Sci., 60, 1420-1428.

Hara, T., and S. E. Belcher, 2002: Wind forcing in the equilibrium range of wind-wave spectra. J. Fluid Mech., 470, 223-245.

$\longrightarrow$, and — 2004: Wind profile and drag coefficient over mature ocean surface wave spectra. J. Phys. Oceanogr., 34, $2345-2358$.

Moon, I.-J., I. Ginis, T. Hara, H. Tolman, C. W. Wright, and E. J. Walsh, 2003: Numerical simulation of sea surface directional wave spectra under hurricane wind forcing. J. Phys. Oceanogr., 33, 1680-1706.

_ , T. Hara, I. Ginis, S. E. Belcher, and H. Tolman, 2004a: Effect of surface waves on air-sea momentum exchange. Part I: Effect of mature and growing seas. J. Atmos. Sci., 61, 23212333.

_ I. Ginis, and T. Hara, 2004b: Effect of surface waves on air-sea momentum exchange. Part II: Behavior of drag coefficient under tropical cyclones. J. Atmos. Sci., 61, 2334-2348.

,-- , and $-2004 \mathrm{c}$ : Effect of surface waves on Charnock coefficient under tropical cyclones. Geophys. Res. Lett., 31, L20302, doi:10.1029/2004GL020988.

,,--- , and B. Thomas, 2007: A physics-based parameterization of air-sea momentum flux at high wind speeds 
and its impact on hurricane intensity predictions. Mon. Wea. Rev., 135, 2869-2878.

Powell, M. D., S. H. Houston, L. R. Amat, and N. MorisseauLeroy, 1998: The HRD real-time hurricane wind analysis system. J. Wind Eng. Ind. Aerodyn., 77-78, 53-64.

, P. J. Vickery, and T. A. Reinhold, 2003: Reduced drag coefficient for high wind speeds in tropical cyclones. Nature, 422, 279-283.

Tolman, H. L., 2002: Validation of WAVEWATCH III version 1.15 for a global domain. NOAA/NWS/NCEP/OMB Tech. Note 213, 33 pp.
- and D. Chalikov, 1996: Source terms in a third-generation wind wave model. J. Phys. Oceanogr., 26, 2497-2518.

_, and J. H. G. M. Alves, 2005: Numerical modeling of wind waves generated by tropical cyclones using moving grids. Ocean Modell., 9, 305-323.

- - - and Y. Y. Chao, 2005: Operational forecasting of wind-generated waves by hurricane Isabel at NCEP. Wea. Forecasting, 20, 544-557.

WAMDI Group, 1988: The WAM model-A third generation ocean wave prediction model. J. Phys. Oceanogr., 18, 17751810 . 